\title{
Editorial
}

\section{Austeridade faz mal à saúde}

I Kenneth Rochel de Camargo Jr. I

Esta é provavelmente a conjuntura mais desfavorável que o SUS já enfrentou desde sua concepção, num momento que agora parece longínquo, há cerca de 30 anos. Embalado por sonhos generosamente utópicos, inscritos no texto constitucional, nosso sistema nacional de saúde sempre enfrentou dificuldades em seu financiamento, mesmo antes de sua formalização legal, quando o Inamps foi repassado para o Ministério da Saúde sem que o respectivo orçamento fosse remanejado.

Com todas as dificuldades, contudo, os governos que se seguiram apoiaram, ainda que parcialmente e com ações ocasionalmente muito contraditórias, a mais importante política de saúde do país. Esse aparente consenso é rompido no presente, quando um ministro da Saúde não hesita em declarar, em entrevista à BBC, que um sistema universal é um "sonho", e seus defensores "ideólogos, não técnicos" - o que chega a ser irônico provindo de um político sem qualificação específica na área que ocupa. Ao mesmo tempo, o mesmo ministro propóe como alternativa a criação de "planos de saúde populares", engodo que não resolverá nenhum dos problemas estruturais da atenção à saúde, mas que certamente representará um tiro certeiro no coração do SUS.

Essa declaração surge no quadro geral de promoção de uma política econômica que tem como base um conceito de austeridade criticado quase que universalmente até por organismos internacionais que até recentemente a apregoavam como virtude, como o FMI e a OCDE. A PEC 55, em tramitação no Senado (anteriormente 241, aprovada na Câmara), tema do comentário desta edição, é outro passo equivocado no sentido da destruição do Estado de BemEstar Social que mal começamos a erigir.

O congelamento por inacreditáveis 20 anos do orçamento público, tomando como base um momento de intensa recessão e retração nas contas públicas, 
ameaça todo um conjunto de políticas públicas, não apenas a Saúde, como também a Educação e a Ciência e Tecnologia, comprometendo qualquer projeto de nação que ainda tivéssemos a esperança de propor.

É importante notar que essas medidas avançam sob o beneplácito de uma imprensa cúmplice, que não dá espaço ao contraditório, apresentando essa política desastrosa como se fosse a única alternativa possível para a crise econômica que atravessamos. Esse pacto de elites em torno de um projeto anti-povo faz lembrar a denúncia de Naomi Klein sobre o "capitalismo de choque" (KLEIN, 2007), que se aproveita de crises (ou as cria) para possibilitar a passagem de medidas impopulares que resultarão inevitavelmente em mais concentração de renda e maior deterioração das condições de vida da maior parte da população.

Fazemos coro à afirmação do presidente da Abrasco, Gastão Wagner Campos, na sua participação no último grande debate do recente Congresso de Ciências Sociais e Humanas em Saúde, realizado em Cuiabá: austeridade faz mal à saúde. ${ }^{2}$

Não nos resta outra alternativa senão resistir, lutar para preservar o SUS e ampliar sua atuação, impedindo sua destruição por interesses econômicos que jamais levaram em consideração a saúde da população. Lembremos que a caminhada para a construção do SUS se iniciou em condições ainda mais adversas do que as que enfrentamos, e ainda assim fomos vitoriosos. Mais que nunca, é fundamental a refundação do Movimento da Reforma Sanitária, como bastiāo de luta por uma política de saúde democrática, equânime, efetiva e justa.

Como em anos anteriores, aproveitamos a última edição do ano para agradecer de público a todos os pareceristas que nos auxiliam na difícil tarefa da editoração científica. Seus nomes estão listados ao final deste fascículo. Adicionalmente, temos feito um agradecimento especial ao parecerista que mais se destacou no ano, pela qualidade e quantidade de suas colaborações. Em 2016 tivemos não apenas um, mas quatro pareceristas a quem devemos um agradecimento especial: Carlos Estellita-Lins, Jairo Gama, Luciana Simas e Vanessa Rangel. A vocês e todos os demais revisores e autores, nosso muito obrigado.

Por fim, saudamos o mais novo acréscimo ao nosso grupo de editores associados: Fernando Vidal, do ICREA, Institució Catalana de Recerca i Estudis Avançats. 


\section{Referência}

KLEIN, N. The shock doctrine: The rise of disaster capitalism. New York: Henry Holt \& Co., 2007.

\section{Notas}

${ }^{1}<$ http://www.bbc.com/portuguese/brasil-37932736>. Acessado em: 01 dez. 2016.

2 https://www.abrasco.org.br/site/2016/10/austeridade-faz-mal-a-saude-diz-o-ultimo-grande-debate-em-cuiaba/ [Acessado em 01/12/2016]

\section{Revisores de 2016}

A todos, nosso agradecimento.

Corpo Editorial e Editores da Physis

Adriana Geisler

Adriane Batiston

Aíla Araújo

Albina Rorres

Alcindo Ferla

Alexandre Grangeiro

Alexandre Ribeiro Wanderley

Alice Uchôa

Aluisio Silva Junior

Ana Pimentel

Ana Canesqui

Ana Cristina Figueiredo

Ana Lucia Heckert

Ana Paula Andrade

Analice Palombini

Andiara Brito

Andre Luis Bonifacio de Carvalho

André Luis Mendonça

André Vital
Anelise Pinheiro

Angela Esher

Ângela Pôrto

Angelica Fonseca

Angelo Roncalli

Anna Uziel

Antonio Augusto Pinto Junior

Antonio Marcos Tosoli Gomes

Antonio Tadeu

Benilton Bezerra

Beth Barros

Bruna Coutinho

Camila Areda

Camilo Venturi

Carlos Estellita-Lins

Carmem Simone Diniz

Ceci Noronha

Celia Pierantoni

Cesar Augusto Favoreto 


Christiana Profice
Cíntia Borges
Claudelí Mistura
Claudia Bonan
Claudia Castro
Claudia Cunha
Claudia Elizabeth Abbês Baeta Neves
Claudia Henschel De Lima
Claudia Mora

Emerson Rasera

Emilia Addison

Enéas Teixeira

Enrique Perdiguero

Erasmo Ruiz

Erika Aragão

Eronildo Felisberto

Erotildes Leal

Eva Zafra

Claudia Osorio-De-Castro

Fabiana Lima

Cleonice Bosa

Fabio Carvalho

Cristian Bonaldi

Fabio Silva

Cristiane Seixas

Fábio Tajra

Cristina Larrea

Fabíola Rohden

Cristineide França

Fatima Siliansky

Dagoberto Pereira

Felipe Asensi

Dalia Montilla

Fernanda Fernandes

Damián Herkovits

Fernando Hellmann

Daniel Costa

Fernando Aith

Daniel Groisman

Fernando Fernandes

Daniela Amaral

Fernando Lefèvre

Daniela Canazaro

Flávia Araújo

Daniela Manica

Flavio Edler

Daniela Murta

Francieli Cembranel

Dannielly Oliveira

Francisco Inácio Bastos

Dario Pasche

Francisco Viacava

Debora Diniz

Franklin Forte

Debora Nitkin

Gabriela Araújo

Deborah Uhr

George Kornis

Denise Barros

Gilberto Hochman

Diane Viana

Gustavo Zambenedetti

Dolors Comas D'Argemir

Hamilton Ferreira Junior

Ediná Costa

Handerson Leite

Edna Granja

Heloisa Barboza

Eduardo Perin

Henrique Salmazo-Silva

Elaine Rabello

Hillegonda Dutilh Novaes

Eliana Costa

Iacã Macerata

Eloisa Grossman

Ideli Domingues

Emerson Merhy

Igor Gomes 
Isabella Samico

Ivia Maksud

Jaime Benchimol

Jairnilson Paim

Jairo Gama

Jane Russo

Janete Naves

Jaqueline Ferreira

João Bastos

João Henrique Scatena

Joao Matos

Joel Lamounier

Jose Pigatto

Jose Canha Dias

José Ferrari

José Maldonado

Josiane Vivian Lima

Juliana Diniz

Juliana Pimenta

Júlio Alencar

Julio Schweickardt

Jussara Reis Prá

Jussara Soares

Karen Andrade

Kellen Rezende

Kellen Santos Rezende

Laia Ventura

Lannuzya Oliveira

Larissa Queiroz

Laura Gonçalves

Laura Lowenkron

Laura Moutinho

Leny Mrech

Leonardo Fontenelle

Leticia Medeiros

Lia Castro

Lidiane Filippin

Lilian Chazan

Liliana Escóssia
Liz Almeida

Lorena Consalter

Luciana Caliman

Luciana Simas

Luciane Lopes

Luciene Rocinholi

Lucio Freitas

Luiz Fadel De Vasconcellos

Luiza Costa

Luiza Jane Eyre Vieira

Luiza Lena

Mabel Gracia Arnaiz

Marcela Aguiar

Marcelo Castellanos

Marcelo Battesini

Marcelo Dalla Vecchia

Marcelo Demarzo

Marcelo Hoexter

Marcelo Kimati

Márcia Alves

Márcia Moraes

Marcia Wagner

Marcio Belloc

Marcio Simeone

Marco Da Ros

Marcos Dias

Maria Cecília Souza Minayo

Maria Delduque

Maria Denise Schimith

Maria Do Carmo Leal

Maria Ines Nemes

Maria Loyola

Maria Paula

Maria Teresa Alves

Maria Veloso De Albuquerque

Mariana Dorsa

Mariangela Cherchiglia

Marilena Correa

Marília Arreguy 
Marismary De Seta

Martin Correa-Urquiza

Martinho Silva

Michelle Sadler

Millani De Almeida

Mirian Ribeiro Baião

Moisés Goldbaum

Monica Nunes

Mônica Angelim

Monica Kornis

Monica Roza

Murilo Mota

Natalia Barbieri

Natalia Fischetti

Nísia Trindade

Nuria Malajovich

Octavio Souza

Paula Cerqueira

Paula Furlan

Paula Gaudenzi

Paula Land

Paula Machado

Paulo César Alves

Paulo Henrique Rodrigues

Pedro Paulo Bicalho

Perla Klautau

Priscila Alves

Rachel Menezes

Rafael Ditterich

Rafael Gomes

Raimunda Silva

Raquel Ramos

Raquel Rigotto

Regina Lucena

Regina Maria Barbosa

Reinaldo Guimaraes
Renato Sampaio

Ricardo Ventura

Rita de Cássia Silva

Rogerio Azize

Ronald Arendt

Rosa Wanda Diez-Garcia

Rosana Onocko Campos

Roseli Shavitt

Rossano Lima

Sabrina Stefanello

Serafim Santos

Siglia Camargo

Silvia Cristina Bocchi

Simone Mainieri Paulon

Simone Monteiro

Sonaly Lima

Stella Taquette

Sued Sarmento

Suely Deslandes

Suzana Canez

Tatiana Lionço

Tatiana Wargas

Tazio Vanni

Teresinha Constantinidis

Thaddeus Blanchette

Túlio Franco

Valdir Oliveira

Vanderlan Silva

Vanessa Rangel

Vera Lucia Pepe

Veriano Terto Jr.

Virgínia Kastrup

Walter Mendes Junior

Willian Santos

Wilza Villela 